\title{
Discriminating new physics scenarios at the Next Linear Collider: The role of polarization
}

\author{
E. M. Gregores \\ Instituto de Física Teórica, Universidade Estadual Paulista, Rua Pamplona 145, 01405-900 São Paulo, Brazil \\ M. C. Gonzalez-Garcia \\ Instituto de Física Teórica, Universidade Estadual Paulista, Rua Pamplona 145, 01405-900 São Paulo, Brazil \\ and Instituto de Física Corpuscular-IFIC/CSIC, Departament de Física Tè̀rica, Universitat de València, \\ 46100 Burjassot, València, Spain \\ S. F. Novaes \\ Instituto de Física Teórica, Universidade Estadual Paulista, Rua Pamplona 145, 01405-900 São Paulo, Brazil
}

(Received 26 March 1997)

\begin{abstract}
We explore the potential of the Next Linear Collider, operating in the $e \gamma$ mode, to disentangle new physics scenarios in single $W$ production. We study the effects related to the exchange of composite fermions in the reaction $e \gamma \rightarrow W \nu_{e}$, and compare them with those arising from trilinear gauge boson anomalous couplings. We stress the role played by the initial state polarization to increase the reach of this machine and to discriminate the possible origin of the new phenomena. [S0556-2821(97)02917-2]

PACS number(s): 12.60.Rc, 13.88.+e, 14.70.-e
\end{abstract}

\section{INTRODUCTION}

The standard model (SM) of the electroweak interactions has received striking confirmation after the recent set of precise measurements made by CERN Large Electron-Positron Collider (LEP) [1]. In particular, the properties of the neutral weak boson and its couplings with fermions were established with great precision. However, similar confidence on other sectors of the SM, such as the vector boson self-couplings which are determined by the non-Abelian gauge structure of the theory, is still lacking. Moreover, the SM does not furnish any reasonable explanation for the replication of fermionic families and their pattern of mass.

In the search for an explanation for the fermionic generations, we face theories where the known particles (leptons, quarks, and gauge bosons) are composite [2], and share common constituents. In this case, the SM should be seen as the low energy limit of a more fundamental theory whose main feature would be the existence of excited states, with mass below or of the order of some large mass scale $\Lambda$.

Searches for composite states have been carried out by several collaborations of the CERN $e^{+} e^{-}$collider LEP [3], and also from DESY electron-proton collider HERA [4]. Recent data from LEP experiments have excluded excited spin$\frac{1}{2}$ electrons with mass up to $80 \mathrm{GeV}$ from the pair production search, and up to $160 \mathrm{GeV}$ from direct single production, for a scale of compositeness $f / \Lambda>0.7 \mathrm{TeV}^{-1}$ (see below). HERA experiments are able to exclude excited electrons with mass below $200 \mathrm{GeV}$ for $f / \Lambda>4.9 \mathrm{TeV}^{-1}$. Bounds on excited electron couplings have also been established through the evaluation of radiative corrections to $Z^{0}$ width at one-loop level [5]. On the theoretical side, there have been extensive studies on the possibility of unravelling the existence of excited fermions in $p p$ [6,7], $e^{+} e^{-}[7-11]$, and ep $[9,10]$ collisions at higher energies.

As pointed out above, another possible source of deviation from the SM predictions, still allowed by experimental results, is the existence of anomalous vector boson selfinteractions. Our knowledge of the structure of the trilinear couplings between gauge bosons remains rather poor despite several experimental searches have been carried out [12]. One of the main goals of LEPII collider at CERN will be the study of the reaction $e^{+} e^{-} \rightarrow W^{+} W^{-}$which will lead to stronger bounds on possible anomalous $W^{+} W^{-} \gamma$ and $W^{+} W^{-} Z$ interactions [13]. There are in the literature several theoretical studies on the probes for these anomalous interactions on future $e^{+} e^{-}[14-16]$ and $p p$ [17] colliders.

An important tool for the search of new physics will be the Next Linear Collider (NLC), an $e^{+} e^{-}$collider that will have a center-of-mass energy of at least $500 \mathrm{GeV}$ with an integrated luminosity around $50 \mathrm{fb}^{-1}$ [18]. At the NLC, it will also be possible to obtain a high energy photon beam via the Compton scattering of a laser off the electron beam $[19,20]$. The so called laser backscattering mechanism will allow to obtain reactions initiated by either $e^{+} e^{-}, e \gamma$, or $\gamma \gamma$ at NLC.

In this work, we establish the optimum strategy to search for excited fermions in the e $\gamma$ mode of the NLC, which is the most promising one for this purpose. The quest for excited leptons can be carried out in all modes of the collider. In $e^{+} e^{-}$collisions, they can be pair produced provided that their mass is below the kinematical threshold. Above threshold the most promising process in $e^{+} e^{-}$is via the $t$-channel contribution to $e^{+} e^{-} \rightarrow \gamma \gamma$. In Ref. [11] it was shown that better limits are obtained from $e \gamma$ collisions, where the excited lepton can be produced as a resonant state in the $s$ channel [11].

This search for excited leptons, however, can be jeopardized by the misidentification of their signal with other possible sources of new phenomena, such as the anomalous gauge boson self-interactions. Therefore, it is important to study how to isolate the excited fermion signal from the one coming from the anomalous triple vector boson coupling. Recent comparison between the reach of NLC, operating in 
the $e^{+} e^{-}$and $e \gamma$ modes [16], shows that both options have comparable sensitivity to the anomalous $W^{+} W^{-} \gamma$ and $W^{+} W^{-} Z$ couplings. One must bear in mind, however, that the limits derived on the $W^{+} W^{-} \gamma$ couplings from the $e^{+} e^{-}$mode are always dependent on further assumptions on the structure of the $W^{+} W^{-} Z$ vertex, as both $W^{+} W^{-} \gamma$ and $W^{+} W^{-} Z$ couplings contribute to the process. In consequence, one cannot rule out from the study of the $e^{+} e^{-}$ mode, the existence of rather large anomalous trilinear gauge couplings to the photon. On the other hand, the $e \gamma$ mode allows to single out the behavior of the $W^{+} W^{-} \gamma$ coupling and to derive a model independent limit.

We analyze here the deviations from the SM predictions for the reactions $e \gamma \rightarrow W \nu_{e}$ at $\sqrt{s_{e e}}=500 \mathrm{GeV}$ due to the exchange of excited spin- $\frac{1}{2}$ fermions, and compare them with the deviations arising from anomalous trilinear gauge boson couplings. We make a detailed study of the experimental signatures of excited fermions and anomalous couplings, exploiting the possibility of polarizing both the electron and laser beams. We design the best strategy to identify the origin of the signal. Our results show that this reaction furnishes stronger limits than the standard reaction $e \gamma \rightarrow e \gamma$ for excited electrons above the kinematical limit. Furthermore, even when the excited electron does not couple to photons, and therefore cannot be produced in the $s$-channel, the existence of the corresponding excited neutrino can be detected in the single $W$ production, via its $t$-channel contribution. We also show how the use of polarization allows the discrimination between the excited lepton signal and the one due to the anomalous trilinear gauge couplings.

The outline of this paper is the following. In Sec. II, we introduce the effective Lagrangians describing the excited fermion and anomalous gauge bosons couplings, and in Sec. III we present the main ingredients of the laser backscattering mechanism with polarized beams. Section IV contains the analysis of the reaction $e \gamma \rightarrow W \nu_{e}$ and displays our results. Finally, in Sec. V, we summarize our conclusions.

\section{EFFECTIVE LAGRANGIANS}

In order to describe the effects of new physics due to both the presence of new excited leptons and to anomalous trilinear couplings we make use of the effective Lagrangian approach. For the excited states, we concentrate in a specific model [9], which has been extensively used by several experimental collaborations $[3,4]$. In doing so, we introduce the weak doublets, for the usual left-handed fermion $\left(\psi_{L}\right)$ and for the excited fermions $\left(\Psi^{*}\right)$, and we write the most general dimension-five effective Lagrangian describing the coupling of the excited fermions to the usual fermions, which is $\mathrm{SU}(2) \times \mathrm{U}(1)$ invariant and $C P$ conserving,

$$
\mathcal{L}_{F f}=\frac{-1}{2 \Lambda} \bar{\Psi}^{*} \sigma^{\mu \nu}\left(g f \frac{\tau^{i}}{2} W_{\mu \nu}^{i}+g^{\prime} f^{\prime} \frac{Y}{2} B_{\mu \nu}\right) \psi_{L}+\text { H.c. },
$$

where $f$ and $f^{\prime}$ are weight factors associated to the $\mathrm{SU}(2)$ and U(1) coupling constants $\left(g\right.$ and $\left.g^{\prime}\right)$, with $\Lambda$ being the compositeness scale, and $\sigma_{\mu \nu}=(i / 2)\left[\gamma_{\mu}, \gamma_{\nu}\right]$. We assume a pure left-handed structure for these couplings in order to comply with the strong bounds coming from the measurement of the anomalous magnetic moment of leptons [21].

In terms of the physical fields, the Lagrangian (1) becomes

$$
\mathcal{L}_{F f}=-\sum_{V=\gamma, Z, W} C_{V F f} \bar{F} \sigma^{\mu \nu}\left(1-\gamma_{5}\right) f \partial_{\mu} V_{\nu}+\text { H.c. },
$$

where $F=N, E$ are the excited leptons, $f=\nu, e$, and $C_{V F f}$ is the coupling of the vector boson with the different fermions,

$$
\begin{gathered}
C_{\gamma E e}=-\frac{e}{4 \Lambda}\left(f+f^{\prime}\right), \\
C_{Z E e}=-\frac{e}{4 \Lambda}\left(f \cot \theta_{W}-f^{\prime} \tan \theta_{W}\right), \\
C_{\gamma N \nu}=\frac{e}{4 \Lambda}\left(f-f^{\prime}\right), \quad C_{Z N \nu}=\frac{e}{4 \Lambda}\left(f \cot \theta_{W}+f^{\prime} \tan \theta_{W}\right), \\
C_{W E \nu}=C_{W N e}=\frac{e}{2 \sqrt{2} \sin \theta_{W} \Lambda} f,
\end{gathered}
$$

where $\theta_{W}$ is the weak angle with $\tan \theta_{W}=g^{\prime} / g$.

For the trilinear gauge coupling, we also write the most general $C$ - and $P$-conserving interaction Lagrangian between the charged gauge bosons and the photon which is $\mathrm{U}(1)_{\mathrm{em}}$ invariant [22]:

$$
\begin{aligned}
\mathcal{L}= & -i e\left[g_{1}^{\gamma}\left(W_{\mu \nu}^{\dagger} W^{\mu} A^{\nu}-W_{\mu}^{\dagger} W^{\mu \nu} A^{\nu}\right)+\kappa_{\gamma} W_{\mu}^{\dagger} W_{\nu} F^{\mu \nu}\right. \\
& \left.+\frac{\lambda_{\gamma}}{M_{W}^{2}} W_{\tau \mu}^{\dagger} W_{\nu}^{\mu} F^{\nu \tau}\right] .
\end{aligned}
$$

For on-shell photons, $g_{1}^{\gamma}=1$ is fixed by electromagnetic gauge invariance since it determines the $W$ electric charge. The coefficients $\kappa(\lambda)$ assumes the values 1(0) in the SM, and are related to the magnetic moment, $\mu_{W}$, and the electric quadrupole moment, $\mathcal{Q}_{W}$, of the $W$ boson, according to

$$
\mu_{W}=\frac{e}{2 M_{W}}\left(1+\kappa_{\gamma}+\lambda_{\gamma}\right) \quad \text { and } \quad \mathcal{Q}_{W}=-\frac{e}{M_{W}^{2}}\left(\kappa_{\gamma}-\lambda_{\gamma}\right) .
$$

In this paper, we are interested in analyzing the influence of both excited fermion and anomalous vector boson coupling in the reaction $e \gamma \rightarrow W \nu_{e}$. The contributions of these new particles and interactions are represented in Fig. 1 as double lines and black dot, respectively.

\section{POLARIZED LASER BACKSCATTERING}

The electron beam of a linear collider can be transformed into a intense photon beam via the process of laser backscattering [19]. The basis of this mechanism lays on the fact that Compton scattering of energetic electrons by soft laser photons results into high energy photons, collimated in the direction of the incident electron.

The backscattered photon distribution function for polarized electron and laser beams can be written [20] as 


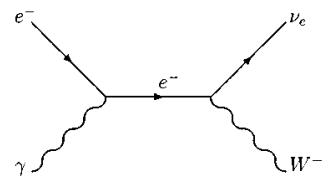

(a)

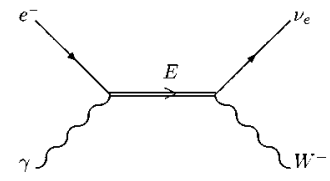

(c)

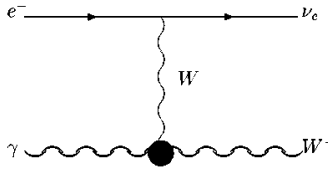

(b)

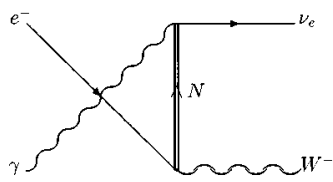

(d)
FIG. 1. Feynman diagrams contributing to the process $e \gamma \rightarrow W \nu_{e}$ in presence of excited fermions (double lines) and anomalous vector boson coupling (black dot).

$$
\begin{aligned}
F\left(x, \zeta ; P_{e}, P_{l}\right)= & \frac{2 \pi \alpha^{2}}{\zeta m^{2} \sigma_{c}}\left[\frac{1}{1-x}+1-x-4 r(1-r)\right. \\
& \left.-P_{e} P_{l} r \zeta(2 r-1)(2-x)\right],
\end{aligned}
$$

where $P_{e}$ is the mean electron longitudinal polarization, $P_{l}$ is the laser photon circular polarization, and $\sigma_{c}$ is the Compton cross section, which can be written as

$$
\sigma_{c}=\sigma_{c}^{0}+P_{e} P_{l} \sigma_{c}^{1},
$$

with

$$
\begin{gathered}
\sigma_{c}^{0}=\frac{2 \pi \alpha^{2}}{\zeta m^{2}}\left[\left(1-\frac{4}{\zeta}-\frac{8}{\zeta^{2}}\right) \ln (\zeta+1)+\frac{1}{2}+\frac{8}{\zeta}-\frac{1}{2(\zeta+1)^{2}}\right], \\
\sigma_{c}^{1}=\frac{2 \pi \alpha^{2}}{\zeta m^{2}}\left[\left(1+\frac{2}{\zeta}\right) \ln (\zeta+1)-\frac{5}{2}+\frac{1}{\zeta+1}-\frac{1}{2(\zeta+1)^{2}}\right] .
\end{gathered}
$$

We have defined the variables

$$
x=\frac{\omega}{E}, \quad \zeta=\frac{4 E \omega_{0}}{m^{2}}, \quad r=\frac{x}{\zeta(1-x)},
$$

where $m$ and $E$ are the electron mass and energy, $\omega_{0}$ is the laser energy and $\omega$ is the backscattered photon energy. The variable $x \leqslant x_{\max } \equiv \zeta /(\zeta+1)$ represents the fraction of the electron energy carried by the back scattered photon. A cutoff value $\zeta=2(1+\sqrt{2}) \simeq 4.83$ is assumed in order to avoid the threshold for electron-positron pair creation through the interaction of the laser and the backscattered photons. The backscattered photon spectrum (5) depends only on the product $P_{e} P_{l}$, and as can be seen from Fig. 2(a), for negative values of this product the spectrum is dominated by hard photons.

A very powerful feature of the Compton backscattering mechanism is the possibility of obtaining a high degree of polarization for the backscattered photons by polarizing the incoming electron and the laser beams. The mean backscattered photon helicity is given by the Stokes parameter
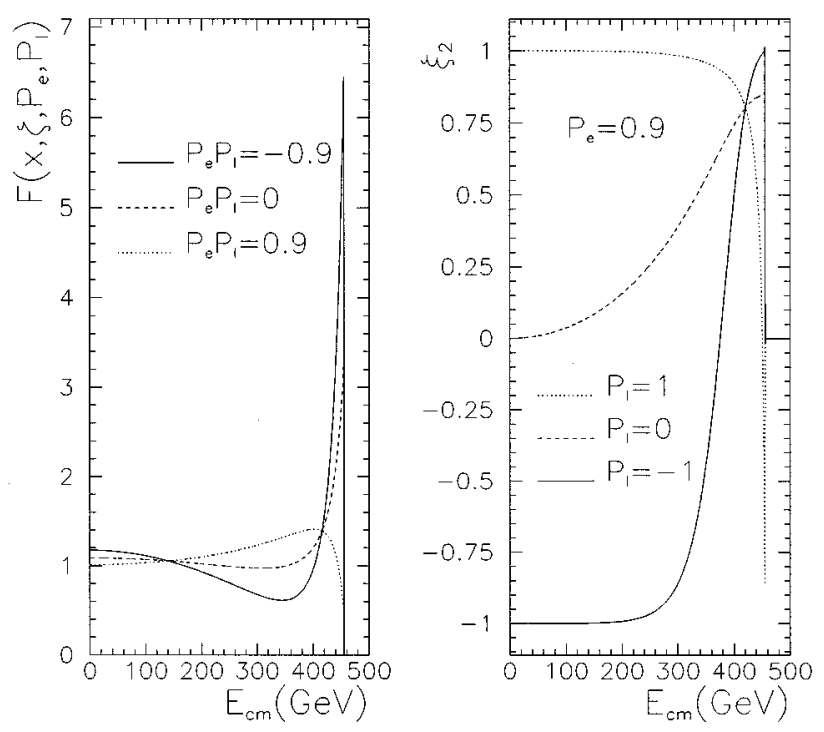

FIG. 2. Photon energy distribution and the circular polarization distribution as a function of the subprocess energy, for different polarization configurations of the electron and laser photon.

$$
\begin{aligned}
\xi_{2}= & \frac{1}{D}\left\{P_{e} r \zeta\left[1+(1-x)(2 r-1)^{2}\right]\right. \\
& \left.-P_{l}(2 r-1)\left[\frac{1}{1-x}+1-x\right]\right\},
\end{aligned}
$$

where

$$
D=\frac{1}{1-x}+1-x-4 r(1-r)-P_{e} P_{l} r \zeta(2 r-1)(2-x) .
$$

For $x=x \max ($ or $r=1)$ and $P_{e}=0$ or $P_{l}= \pm 1$, we have $\xi_{2}=-P_{l}$, i.e., the polarization of the backscattered photon beam has the opposite value of the laser polarization. One can also see that for $x=\zeta /(\zeta+2)$ (or $r=1 / 2)$ the Stokes parameter $\xi_{2}$ is independent of the laser polarization [see Fig. 2(b)], and is given by

$$
\xi_{2}^{(r=1 / 2)}=P_{e} \frac{\zeta(\zeta+2)}{\zeta(\zeta+2)+4} .
$$

The cross section for the reaction $\gamma e \rightarrow X$ in an electronpositron linear collider where the positron beam, with longitudinal polarization $P_{p}$, is converted into a backscattered photon beam, is given by

$$
d \sigma_{P_{e} \xi_{2}}(\gamma e \rightarrow X)=\kappa \int_{x_{\min }}^{x_{\max }} d x F\left(x, \zeta ; P_{p}, P_{l}\right) d \hat{\sigma}_{P_{e} \xi_{2}}(e \gamma \rightarrow X)
$$

where $\kappa$ is the efficiency of the laser conversion of the electrons into photons and $d \hat{\sigma}_{P_{e} \xi_{2}}$ is the polarized cross section for the subprocess $\gamma e \rightarrow X$, which is a function of $\hat{s}=x s$. In our calculation we assume that $100 \%$ of the electrons are converted into photons $(\kappa=1)$.

The polarized subprocess cross section can be written as 


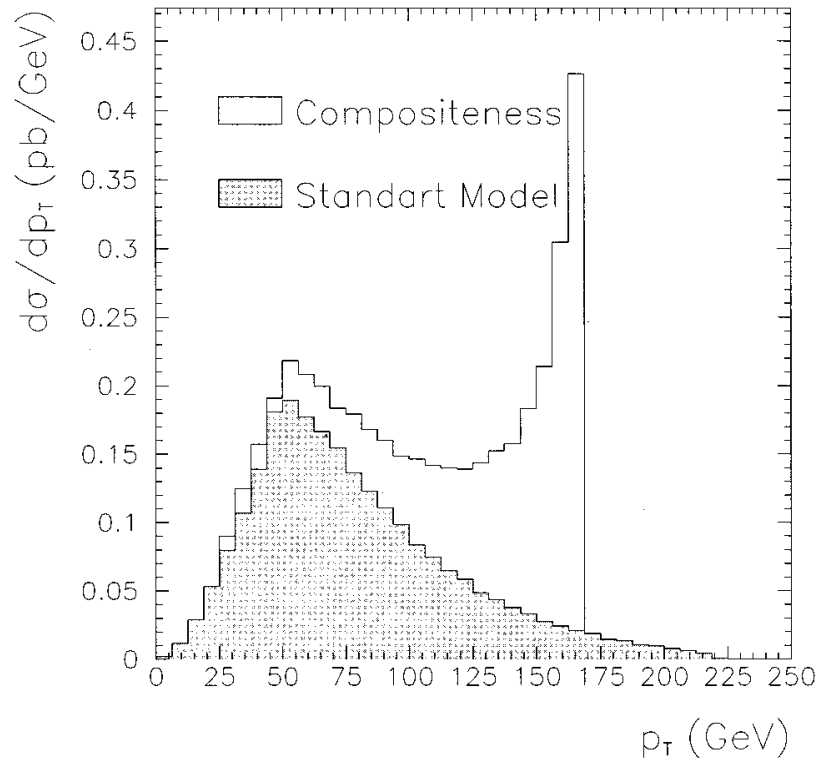

FIG. 3. Tranverse momentum distribution of $W$ bosons in the presence of an excited electron with $M_{E}=350 \mathrm{GeV}$, compared to the SM prediction, for $f=f^{\prime}=1$, and $\Lambda=1 \mathrm{TeV}$.

$$
\begin{aligned}
d \hat{\sigma}_{P_{e} \xi_{2}}= & \frac{1}{4}\left[\left(1+P_{e} \xi_{2}\right)\left(d \hat{\sigma}_{++}+d \hat{\sigma}_{--}\right)\right. \\
& +\left(P_{e}+\xi_{2}\right)\left(d \hat{\sigma}_{++}-d \hat{\sigma}_{--}\right)+\left(1-P_{e} \xi_{2}\right)\left(d \hat{\sigma}_{+-}\right. \\
& \left.\left.+d \hat{\sigma}_{-+}\right)+\left(P_{e}-\xi_{2}\right)\left(d \hat{\sigma}_{+-}-d \hat{\sigma}_{-+}\right)\right]
\end{aligned}
$$

with $d \hat{\sigma}_{\lambda_{e} \lambda_{\gamma}}\left(\lambda_{e(\gamma)}= \pm 1\right)$ being the polarized subprocess cross section for full electron and photon polarization, $P_{e}$ is the longitudinal polarization of the electron beam, and the Stokes parameter, $\xi_{2}$, is given in Eq. (9).

\section{RESULTS}

\section{A. Excited fermions signature}

The standard mechanism to establish the existence of an excited electron with mass below the kinematical reach of the $e \gamma$ machine is the identification of the Breit-Wigner profile in the $e \gamma$ invariant mass distribution of the process $e \gamma \rightarrow e \gamma[11]$. This is obviously only possible when the excited electron couples to the photon, i.e., $f \neq-f^{\prime}$ [see Eq. (3)]. On the other hand, the reaction $e \gamma \rightarrow W \nu_{e}$ is sensitive to both the exchange of the excited electron in the $s$ channel and to the exchange of the excited neutrino in the $t$ channel (see Fig. 1). The characteristic signature of the excited leptons will therefore depend on the excited fermion mass and on the relative weight of the $s$-channel versus the $t$-channel contribution or, in other words, the relative sizes of $f$ and $f^{\prime}$.

As in the reaction $e \gamma \rightarrow e \gamma$, the existence of an excited electron with nonvanishing coupling to the photon and with mass below the kinematical reach of the $e \gamma$ collider, can also be easily identified in the reaction $e \gamma \rightarrow W \nu_{e}$ but now through the study of the $W$ transverse momentum distribution, $d \sigma / d p_{T}$. For illustration, we present in Fig. 3 the $p_{T}$ distribution of the $W$ produced in the reaction $e \gamma \rightarrow W \nu_{e}$, for
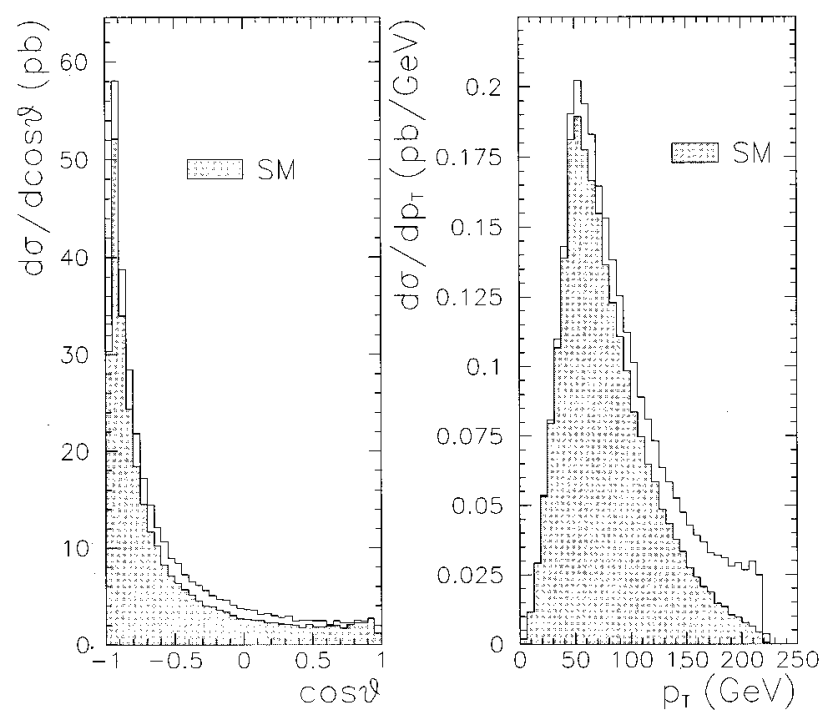

FIG. 4. $W$ boson kinematical distributions in the presence of composite states, compared to the SM predictions, for $f=f^{\prime}=1$, and $M_{E}=\Lambda=500 \mathrm{GeV}$.

$M_{E}=350 \mathrm{GeV}$ at an $e^{+} e^{-}$collider with $\sqrt{s}=500 \mathrm{GeV}$. We introduced a cut of $15^{\circ}$ in the polar angle $(\theta)$ of the detectable final state particles with the beam pipe to ensure their detection. We also assumed a reconstruction efficiency of $60 \%$ for the produced $W^{\prime}$ 's. As expected, the existence of excited states with mass below the kinematical reach of the e $\gamma$ collider provides an very clear signal, the Jacobian peak at $p_{T} \sim M_{E} / 2$. This situation will be no longer considered here, since our main concern is the possibility of misidentification of new physics effects on the NLC, and in this scenario, the existence of excited fermions can easily be set apart from the anomalous vector boson contribution.

For excited leptons above the kinematical limit of the collider we still find an enhancement on the total cross section due to the $s$-channel contribution. We also find an effect in the distribution of the produced $W$. We have simulated these distributions for $M_{E}=\Lambda=500 \mathrm{GeV}$ and $f=f^{\prime}$. We present on Fig. 4 the transverse momentum distribution, and the angle between the $W$ and the electron beam direction for unpolarized beams. As seen in the figure, the existence of excited fermions leads to an enhancement of $W$ production at large $p_{T}$ which reflects the tail of the jacobian peak. In the angular distribution of the $W$ the effect of composition is very small.

The process mediated by the exchange in the $t$-channel of excited neutrinos coupled to photons takes place when $f$ $\neq f^{\prime}$, and gives a much smaller effect. This process is particularly interesting in the extreme situation when $f=-f^{\prime}$ since, in this case, $C_{\gamma E e}$ vanishes, and just the $t$-channel diagram contributes. This contribution gives rise to a destructive interference which diminishes the total $W$ yield, without altering the shape of the transverse momentum and angular distributions in a significant way (see Fig. 5).

In order to estimate the reach of an $e \gamma$ collider to search for new physics, we defined the statistical significance of the signal $(\mathcal{S})$ as 

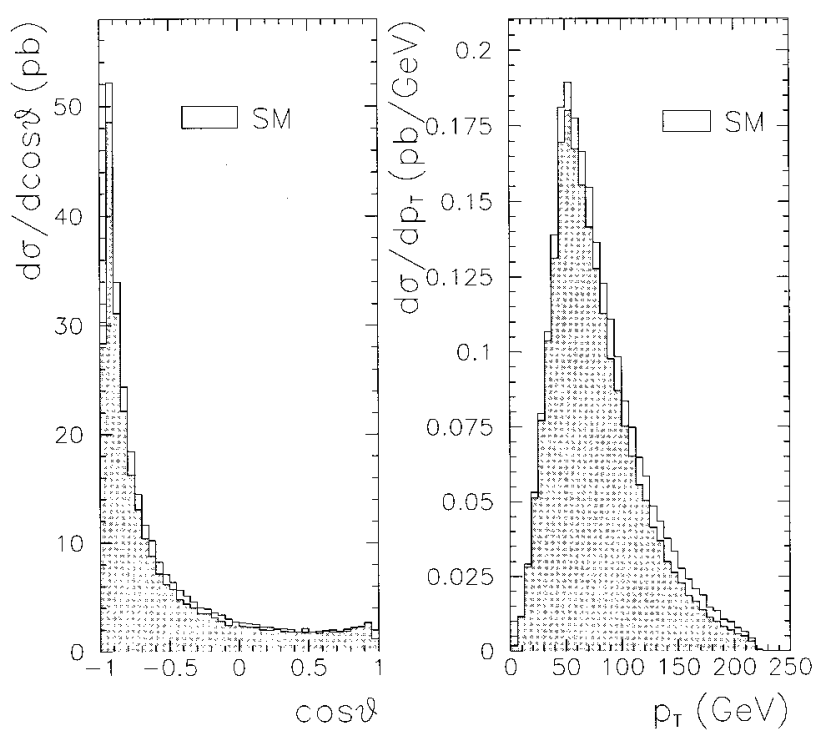

FIG. 5. The same as Fig. 4, for $f=f^{\prime}=-1$, and $M_{N}=\Lambda=300 \mathrm{GeV}$.

$$
\mathcal{S} \equiv \frac{\left|\sigma_{\text {new }}-\sigma_{\mathrm{SM}}\right|}{\sqrt{\overline{\sigma_{\mathrm{SM}}}}} \sqrt{\mathcal{L}_{\text {ee }}}
$$

where $\sigma_{\text {new }}\left(\sigma_{\mathrm{SM}}\right)$ is the total cross section for new physics (standard model) contributions, and $\mathcal{L}_{\mathrm{ee}}$ is the integrated luminosity of the machine. We will assume $\mathcal{L}_{\mathrm{ee}}=50 \mathrm{pb}^{-1}$ for the NLC. In order to ensure that the event is well within the detector volume, we restrict the polar angle of all detectable final particles with the beam pipe to be smaller than $15^{\circ}$ $(|\cos \theta| \leqslant 0.97)$. With this requirement, we assumed that $60 \%$ of the produced $W$ 's can be properly reconstructed.

In Fig. 6, we show the discovery limits for the composite state in the $\Lambda \times M_{E}$ plane for both $e \gamma$ and $W \nu_{e}$ final states, requiring a $3 \sigma$ effect in the total cross section. For unpolar-

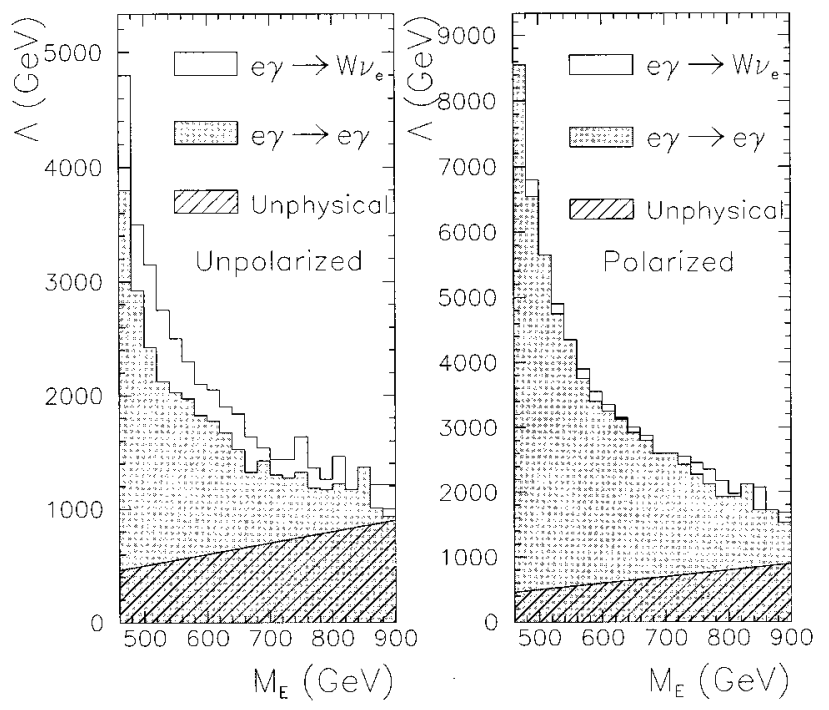

FIG. 6. Discovery contour for $f=f^{\prime}=1$. Inside the shaded regions the deviations from $\mathrm{SM}$ are greater $3 \sigma$. We excluded the unphysical region where $\Lambda<M_{E}$.

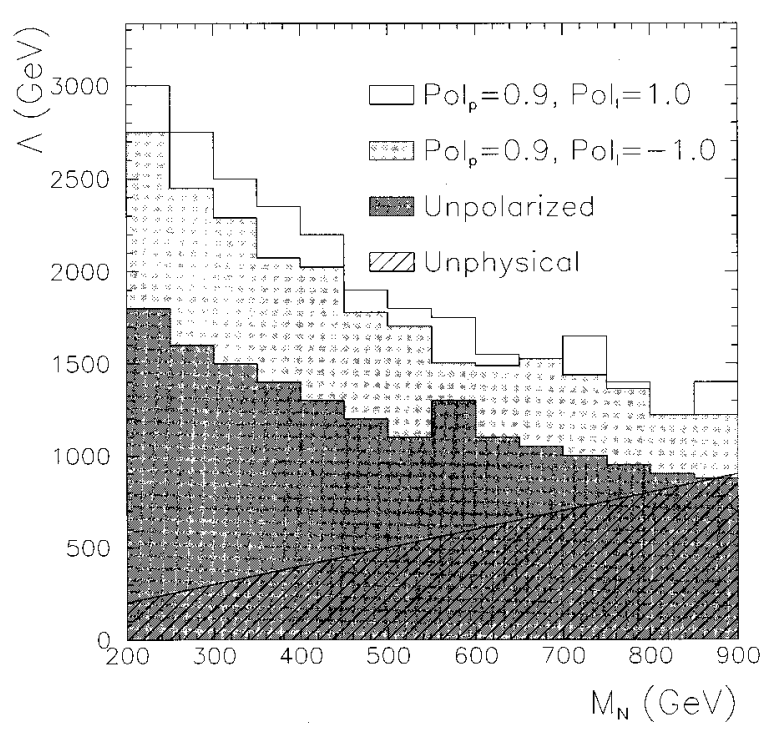

FIG. 7. Discovery contour for composite neutrinos, for $f=-f^{\prime}$. Inside the shaded regions, deviations from SM are greater than $3 \sigma$. We excluded the unphysical region where $\Lambda<M_{N}$.

ized photons the $e W$ channel leads to stronger limits than the e $\gamma$ channel which has large background coming from Compton scattering. However an excess in the $e \gamma$ production is a definite signal of compositeness, since there is no contribution of anomalous couplings in this channel. Moreover, as mentioned above, if the weight factors $f$ and $f^{\prime}$ are such that $f=-f^{\prime}$, the signal of composition on the $e \gamma$ production will disappear completely, but survive on single $W$ production through the exchange of a neutral composite fermion on the $t$ channel. The discovery limit of the NLC for such neutral states is shown in Fig. 7.

Polarization can be used to improve the discovery region in the $\Lambda \times M$ plane through the enhancement of the luminosity and the cross section. The photon distribution functions assume approximately the same value at $\bar{x}=\zeta /(\zeta+2)$ $\simeq 0.71$, even for different polarization configurations of the initial particles (see Fig. 2). In the interval $0<x<\bar{x}$, the luminosity is higher for $P_{p} P_{l}>0$, whereas for the range $x>\bar{x}$ the distribution with $P_{p} P_{l}<0$ dominates. Therefore, in order to search for excited electrons with mass above $\overline{M_{E}}=\sqrt{\overline{x s}}$, we should employ the polarization configurations of the positron and the laser in such a way that $P_{p} P_{l}<0$. The degree of circular polarization of the scattered photon, $\xi_{2}$, has the same sign as the positron polarization in the region of interest. Because of the chiral nature of the weak interactions, just left-hand electrons will produce $W$ 's, and therefore, only electrons and photons with negative helicity contribute to the exchange of an excited electron in the $s$ channel. In this case, the best strategy is to require that the electron and positron have negative polarization, while the laser is set up with positive polarization. We assume that $90 \%$ of beam polarization is achievable at the NLC and that the laser can be $100 \%$ polarized (i.e., $P_{e^{-}}=P_{e^{+}}=-0.9$, and $\left.P_{l}=1\right)$. With this setup the discovery region can be enlarged as much as shown on Fig. 6.

The situation changes when we are dealing with the process involving the excited neutrino. As before, just left- 

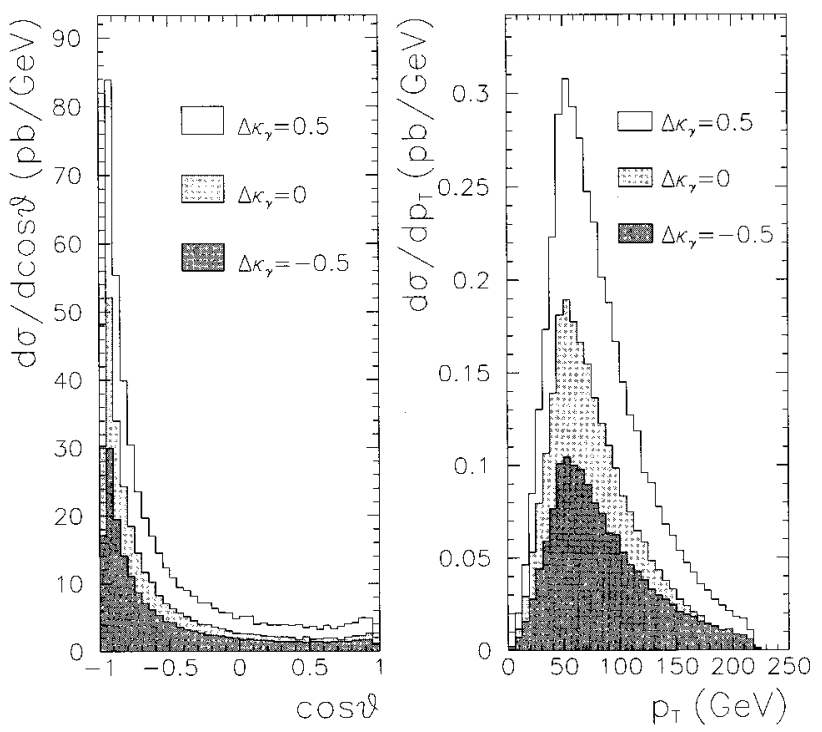

FIG. 8. Effects of a variation on $\Delta \kappa_{\gamma}$ in $W$ kinematical distributions, compared with the standard model $\left(\Delta \kappa_{\gamma}=0\right)$.

handed electrons will participate in the reaction and again one must choose $P_{e^{-}}<0$, but now the photon line is attached to the final state neutrino, and consequently, just the positive helicity photons couple to the fermionic line. According to the discussion above, to obtain a highly energetic and positively polarized photon beam, we must set the positron polarization to be positive $\left(P_{e^{+}}=0.9\right)$ and the laser polarization to be negative $\left(P_{l}=-1\right)$. However, one must also notice that in this process, when the positron and laser have positive polarization, most of the photons have positive helicity (except the very high energy ones) while, at the same time, the SM background coming from the diagram with self-boson interaction is reduced. Due to this reduction, the configuration $P_{e^{-}}=-P_{e^{+}}=-0.9$ and $P_{l}=1$ leads to better limits as can be seen in Fig. 7.

\section{B. Anomalous gauge boson couplings: Comparison}

Let us now examine the consequences of the existence of trilinear anomalous couplings in the single $W$ production. In order to clarify the effect of each of the two possible anomalous couplings $\left(\Delta \kappa_{\gamma}\right.$ and $\left.\lambda_{\gamma}\right)$, we envisaged two distinct scenarios, by keeping just one of them different from zero at a time. In Fig. 8, we show the angular and transverse momentum distributions for different values of $\Delta \kappa_{\gamma}=1-\kappa_{\gamma}$ while keeping $\lambda_{\gamma}=0$. We can see from these figures that the only effect of varying $\Delta \kappa_{\gamma}$ is to increase or decrease the total cross section depending on its sign, while producing small effect on the shape of the distributions. The number of observed events is larger (smaller) than the SM expectation for $\Delta \kappa_{\gamma}>0\left(\Delta \kappa_{\gamma}<0\right)$.

Conversely, in Fig. 9, we show the angular and transverse momentum distributions for different values of $\lambda_{\gamma}$ while keeping $\Delta \kappa_{\gamma}=0$. We can see in these figures that the cross section is always larger than the SM prediction for any sign of the coupling $\lambda_{\gamma}$. The presence of a nonvanishing $\lambda_{\gamma}$ also increases the number of $W$ 's produced in the central and forward direction and of those produced with high $p_{T}$.
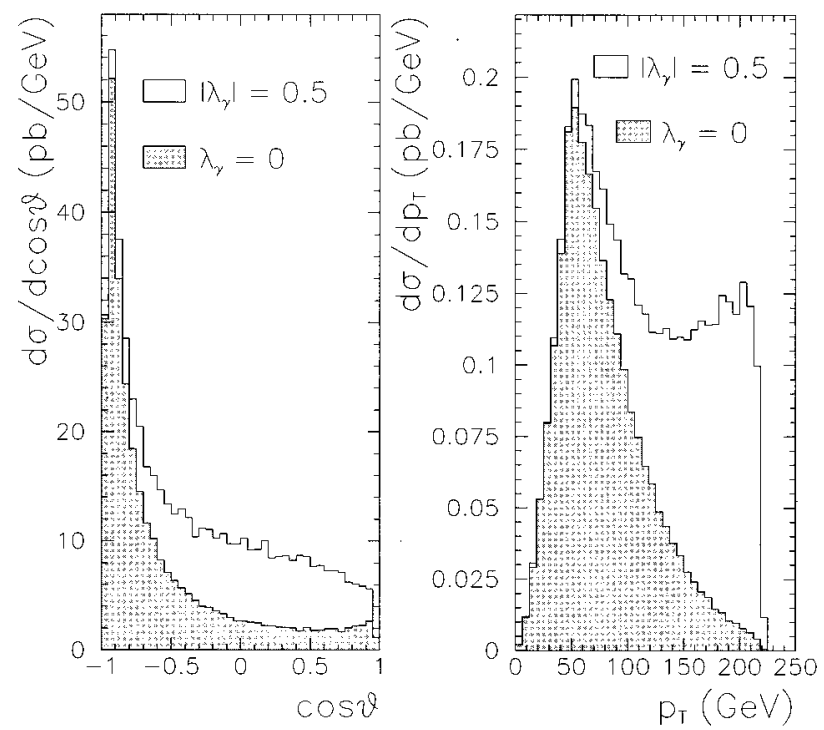

FIG. 9. Effects of a variation on $\lambda_{\gamma}$ in the $W$ distributions, compared with the standard model $\left(\lambda_{\gamma}=0\right)$.

Polarization has also proven to be very useful to unravel the existence of anomalous couplings, and the discovery limits for anomalous couplings have been extensively covered elsewhere [15]. In what follows we will concentrate on the possibility of distinguishing these effects from those arising from the existence of the excited leptons.

Let us now discuss the different scenarios we can face when the NLC starts its operation. It will probably start on its unpolarized mode, in order to be as "democratic" as possible to discover new physics. The first observable that the accumulated statistics will allow to measure is the total cross section. From what we saw above, the effect of new physics can either increase or decrease the total $W$ production.

If the number of produced $W$ 's is greater than expected, one should just look into the $e \gamma$ events that will be produced at the same time. As we saw, in the framework where composition leads to an enhancement on the total single $W$ cross section, the $e \gamma$ channel is also very sensitive to the presence of excited leptons. An increase in the $e \gamma$ cross section would indicate that the excess of $W$ is due to the existence of an excited electron. If no excess is seen in the $e \gamma$ channel, likelihood fittings to the angular and transverse momentum distributions will be able to determine the anomalous coupling parameters leading to such deviations.

On the other hand, if the total cross section is smaller than predicted by the SM, two possibilities remain: (i) the trilinear coupling is anomalous with the value of $\Delta \kappa_{\gamma} \leqslant 0$, or, (ii) the excited neutrino exists, and its gauge structure is such that $f \simeq-f^{\prime}$. To tell these two possibilities apart, we define the polarization asymmetry

$$
A_{\mathrm{pol}}=\frac{\Delta \sigma_{+-}-\Delta \sigma_{-+}}{\Delta \sigma_{+-}+\Delta \sigma_{-+}},
$$

where, for instance,

$$
\Delta \sigma_{+-}=\sigma_{+-}^{\mathrm{SM}}-\sigma_{+-}^{\mathrm{obs}}
$$



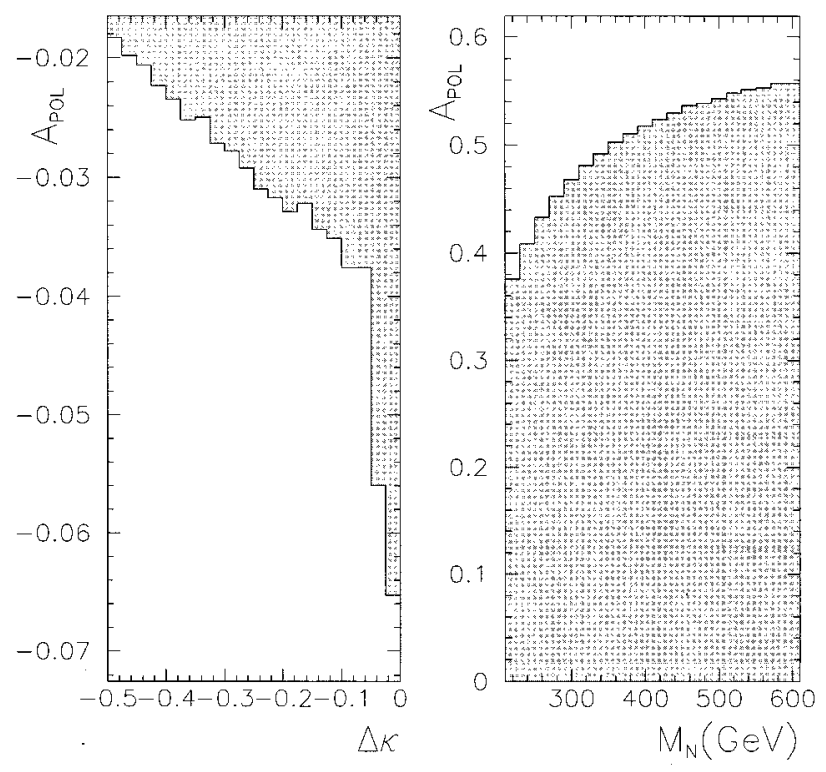

FIG. 10. Polarization asymmetry due to the presence of anomalous couplings with $\Delta \kappa_{\gamma} \leqslant 0$ and in models with excited neutrinos for $f=-f^{\prime}=1$, and $M_{N}=\Lambda$.

measures the deviation from the SM prediction when the positron and laser polarizations are set $P_{e^{+}}=0.9$ and $P_{l}=-1$, always keeping $P_{e^{-}}=-0.9$. Correspondingly, $\Delta \sigma_{-+}$measures the deviation from the SM prediction for $P_{e^{+}}=-0.9$ and $P_{l}=1$.

In Fig. 10, we plot $A_{\text {pol }}$ for the two type of models here considered. As seen in this figure, the asymmetry due to the presence of anomalous trilinear gauge couplings $\lambda_{\gamma}=0$ and $\Delta \kappa_{\gamma} \leqslant 0$ is always negative and very small. On the contrary, the presence of excited neutrinos would yield a larger positive asymmetry. This occurs because photons with both polarizations contribute to the anomalous $\Delta \kappa_{\gamma}$ term, while only positive helicity photons enter in the excited neutrino contribution. Therefore in both configurations the reduction in the cross section due to the negative $\Delta \kappa_{\gamma}$ is of the same order and the corresponding asymmetry is small. However in the configuration $(+-)$, since most of the high energy photons have positive helicity, the effect of the excited neutrino contribution is enhanced and the destructive interference is larger. Consequently $\Delta \sigma_{+-} \gg \Delta \sigma_{-+}$, what gives a positive and larger asymmetry.

\section{CONCLUSION}

In this work, we have studied the deviations from the SM predictions for the reactions $e \gamma \rightarrow W \nu_{e}$ at $\sqrt{s_{e e}}=500 \mathrm{GeV}$ due to two possible sources of new physics. We have analyzed the effect associated to the existence of excited spin$\frac{1}{2}$ fermions, and we have compared them to those arising from anomalous trilinear couplings between the gauge bosons. We have discussed how the use of polarization can improve the reach of the machine in the search for excited fermions. Our results show that this reaction can furnish stronger limits than the standard reaction $e \gamma \rightarrow e \gamma$ for excited electrons above the kinematical limit. We have shown how, by setting up the electron, positron, and laser polarizations, we can be sensitive to scales, $\Lambda$, up to $9 \mathrm{TeV}$, which is twice the bound obtained just with unpolarized beams. Our results show that excited electrons coupling to photons with strength $e$ can be ruled out for $M_{E} \leqslant 1 \mathrm{TeV}$. In addition, the simultaneous analysis of both $e \gamma$ and $W \nu_{e}$ channels allows to discriminate the excited electron signature from the one due to the presence of anomalous trilinear gauge couplings which would lead to the same increase in the total $W$ yield.

Single $W$ production is also the main channel to look for excited neutrinos when the corresponding excited electron does not couple to photons. In this case, we get a reduction in the number of events, as compared to the SM prediction, due to the destructive interference between the SM contribution and the one due to the exchange of the excited neutrino in the $t$ channel. This reduction is significative enough to rule out excited neutrinos with $M_{N} \leqslant 1 \mathrm{TeV}$. Anomalous trilinear couplings with very small $\lambda_{\gamma}$ and $\Delta \kappa_{\gamma} \leqslant 0$ would also lead to a decrease in the number of events which could fake the existence of an excited neutrino. For this case we have introduced a polarization asymmetry which is sensitive to the origin of the deviation.

\section{ACKNOWLEDGMENTS}

M.C. Gonzalez-Garcia is grateful to the Instituto de Física Teórica of the Universidade Estadual Paulista for its kind hospitality. This work was supported by Fundação de Amparo à Pesquisa do Estado de São Paulo, by DGICYT under Grant No. PB95-1077, by CICYT under Grant No. AEN961718, and by Conselho Nacional de Desenvolvimento Científico e Tecnológico.
[1] The LEP Collaborations ALEPH, DELPHI, L3, OPAL, and the LEP Electroweak Working Group, contributions to the 28th International Conference on High-Energy Physics (ICHEP 96), Warsaw, Poland, 1996 [Report No. CERN-PPE/ 96-183, 1996 (unpublished)].

[2] For a review, see, for instance, H. Harari, Phys. Rep. 104, 159 (1984); M. E. Peskin, in Proceedings of the International Symposium on Lepton and Photon Interactions at High Energies, Kyoto, Japan, 1985, edited by M. Konuma and K. Takahashi (RIFP, Kyoto, 1986), p. 714; H. Terazawa, in Proceedings of the XXII International Conference on High Energy Physics, Leipzig, Germany, 1984, edited by A. Meyer and E. Wieczorek (Akademic derwissenschaften der OCR Zeipten, 1984), p. 63; W. Buchmüller, Acta Phys. Austriaca Suppl. 27, 517 (1985).

[3] ALEPH Collaboration, D. Buskulin et al., Phys. Lett. B 385, 445 (1996); OPAL Collaboration, K. Ackerstaff et al., ibid. 391, 197 (1997); DELPHI Collaboration, P. Abreu et al., ibid. 393, 245 (1997); L3 Collaboration, M. Acciarri et al., Report No. CERN-PPE-97-012, 1997 (unpublished). 
[4] H1 Collaboration, I. Abt et al., Nucl. Phys. B483, 44 (1997); ZEUS Collaboration, M. Derrick et al., Z. Phys. C 65, 627 (1995).

[5] M. C. Gonzalez-Garcia and S. F. Novaes, Nucl. Phys. B486, 3 (1997).

[6] K. Enqvist and J. Maalampi, Phys. Lett. 135B, 329 (1984).

[7] J. Kühn and P. Zerwas, Phys. Lett. 147B, 189 (1984).

[8] N. Cabibbo, L. Maiani, and Y. Srivastava, Phys. Lett. 139B, 459 (1984); F. A. Berends and P. H. Daverveldt, Nucl. Phys. B272, 131 (1986); A. Feldmaier, H. Salecker, and F. C. Simm, Phys. Lett. B 223, 234 (1989); M. Martinez, R. Miquel, and C. Mana, Z. Phys. C 46, 637 (1990); F. Boudjema and A. Djouadi, Phys. Lett. B 240, 485 (1990); Maria BardadinOtwinowska, Z. Phys. C 55, 163 (1992); J. C. Montero and V. Pleitez, Phys. Lett. B 321, 267 (1994).

[9] K. Hagiwara, S. Komamiya, and D. Zeppenfeld, Z. Phys. C 29, 115 (1985).

[10] F. Boudjema, A. Djouadi, and J. L. Kneur, Z. Phys. C 57, 425 (1993).

[11] I. F. Ginzburg and D. Yu. Ivanov, Phys. Lett. B 276, 214 (1992); T. Kon, I. Ito, and Y. Chikashige, ibid. 287, 277 (1992); E. Boos, A. Pukhov, and A. Beliaev, ibid. 296, 452 (1992); O. J. P. Éboli, E. M. Gregores, J. C. Montero, S. F. Novaes, and D. Spehler, Phys. Rev. D 53, 1253 (1996).

[12] CLEO Collaboration, M. S. Alam et al., Phys. Rev. Lett. 74, 2885 (1995); CDF Collaboration, F. Abe et al., ibid. 74, 1936 (1995); D0 Collaboration, S. Abachi et al., ibid. 75, 1023 (1995).

[13] G. Gounaris et al., in Physics at LEP2, edited by G. Altarelli, T. Sjöstrand, and F. Zwirner (CERN, Geneva, 1996), Vol. 1, p. 525, hep-ph/9601233.

[14] $e^{+} e^{-}$Collisions at $500 \mathrm{GeV}$ : The Physics Potential (Proceed- ings of the Workshop, Munich, Annecy, Hamburg, 1991, edited by P. Zerwas (DESY Report No. 93-123C, Hamburg, 1993).

[15] E. Yehudai, Phys. Rev. D 41, 33 (1990); S. Choi and F. Schrempp, Phys. Lett. B 272, 149 (1991); S. J. Brodsky, T. Rizzo, and I. Schmidt, Phys. Rev. D 52, 4929 (1995); K. Cheung, S. Dawson, T. Han, and G. Valencia, ibid. 51, 5 (1995).

[16] A. A. Likhoded et al., Report No. IC/93/288, and hep-ph/9309322 (unpublished); M. Raidal, Nucl. Phys. B441, 49 (1995).

[17] H. Aihara et al., "Summary of the Working Subgroup on Anomalous Gauge Boson Interactions of the DPF Long-Range Planning Study,', to be published in Electroweak Symmetry Breaking and Beyond the Standard Model, edited by T. Barklow, S. Dawson, H. Haber, and J. Seigrist (Report No. hep-ph/9503425).

[18] See, for instance, the Next Linear Collider R\&D Homepage, http://nlc.physics.upenn.edu/nlc/nlc.html, and the NLC Zeroth-Order Design Report at http:// www.slac.stanford.edu/accel/nlc/zdr/

[19] F. R. Arutyunian and V. A. Tumanian, Phys. Lett. 4, 176 (1963); R. H. Milburn, Phys. Rev. Lett. 10, 75 (1963); I. F. Ginzburg, G. L. Kotkin, V. G. Serbo, and V. I. Telnov, Nucl. Instrum. Methods Phys. Res. 205, 47 (1983); V. I. Telnov, Nucl. Instrum. Methods Phys. Res. A 294, 72 (1990).

[20] I. F. Ginzburg, G. L. Kotkin, V. G. Serbo, and V. I. Telnov, Nucl. Instrum. Methods Phys. Res. A 219, 5 (1984).

[21] S. J. Brodsky and S. D. Drell, Phys. Rev. D 22, 2236 (1980); F. M. Renard, Phys. Lett. 116B, 264 (1982).

[22] K. Hagiwara, R. D. Peccei, D. Zeppenfeld, and K. Hikasa, Nucl. Phys. B282, 253 (1987). 